\title{
Breast cancer-associated macrophages promote tumorigenesis by suppressing succinate dehydrogenase in tumor cells $\pi$
}

\author{
Valentí Gómez ${ }^{1,}{ }^{*}$, Thomas R. Eykyn², Rami Mustapha ${ }^{3}$, Fabián Flores-Borja $^{4}$, Victoria Male ${ }^{5}$, Paul R. \\ Barber $^{1}$, Antonia Patsialou${ }^{1}$, Ryan Green ${ }^{4}$, Fani Panagaki ${ }^{2}$, Chun W. Li ${ }^{2}$, Gilbert O. Fruhwirth², Susana \\ Ros $^{6}$, Kevin M. Brindle ${ }^{6}$ and Tony $\mathrm{Ng}^{1,3,4,}{ }^{*}$
}

${ }^{1}$ UCL Cancer Institute, University College London, London, WC1E 6DD, UK.

${ }^{2}$ School of Biomedical Engineering \& Imaging Sciences, King's College London, London, SE1 7EH, UK.

${ }^{3}$ School of Cancer and Pharmaceutical Sciences, King's College London, London, SE1 1UL, UK.

${ }^{4}$ KCL Breast Cancer Now Research Unit, Department of Research Oncology, Guy's Hospital, King's College London, London, SE1 1UL, UK.

${ }^{5}$ Institute of Immunity and Transplantation, Royal Free Hospital, University College London, London, NW3 2QG, UK.

${ }^{6}$ Cancer Research UK - Cambridge Institute, University of Cambridge, Li Ka Shing Centre, Cambridge, CB2 ORE, UK.

${ }^{*}$ Corresponding author. Email: tony.ng@kcl.ac.uk (T.N.), valentin.gomez@ucl.ac.uk (V.G.)

TThis manuscript has been accepted for publication in Science Signaling. This version has not undergone final editing. Please refer to the complete version of record at https://eur03.safelinks.protection.outlook.com/?url=http\%3A\%2F\%2Fwww.sciencesignaling.org\%2 F\&amp;data $=01 \% 7$ C01\%7Ctony.ng\%40kcl.ac.uk\%7C2d19339cd8984cadaa7108d849f897c2\%7C837 0cf1416f34c16b83c724071654356\%7C0\&amp;sdata=cpifq2B4nCbBHEzUAWzK323U7pxgL4alofb5F 89EtHs\%3D\&amp;reserved $=0$. The manuscript may not be reproduced or used in any manner that does not fall within the fair use provisions of the Copyright Act without the prior, written permission of AAAS. 


\section{ABSTRACT}

Various cells in the tumor microenvironment are implicated in breast cancer progression. Tumor-associated macrophages (TAMs) suppress antitumor immune responses and enhance migration, invasion, intravasation and subsequent metastasis. We found that the antiinflammatory polarization of TAMs induced metabolic changes in breast cancer cells that supported their growth and metastasis. Notably downregulating the expression of succinate dehydrogenase (SDH) upon increased activity of the TGF $\beta$ signaling pathway and subsequent reduced expression of the STAT1 transcription factor. Anti-inflammatory TAMs induced a decrease in SDH levels resulted in an accumulation of succinate in tumor cells, which in turn promoted the stabilization of the transcription factor HIF1 $\alpha$. Therefore, hypoxic activity reprogrammed cancer metabolism to create a glycolytic state. In a 4T1 mouse model, TAM depletion-repletion experiments revealed that anti-inflammatory macrophages promoted accumulation of glycolysis-derived metabolites, HIF-associated vascularization and expression of the immuno-suppressive protein PD-L1 in tumors. The findings suggest that anti-inflammatory-state TAMs promote tumor growth, angiogenesis and immunosuppression by altering metabolism in breast cancer cells. 


\section{INTRODUCTION}

Dysregulation of cellular metabolism constitutes one of the hallmarks of tumor progression (1). Due to their high proliferative rate, cancer cells have a high demand for both energy (adenosine triphosphate; ATP) and biosynthetic precursors, as well as a need to counter the impact of increased production of reactive oxygen species (ROS) (2). Cancer cells are characterized by a range of genetic adaptations that include the activation of oncogenes and the inhibition of tumor suppressors. Specific gain or loss of function mutations that alter enzyme activity or upregulation of transcription factors that lead to altered protein expression and re-routing of metabolism are also commonly observed in cancer. One such adaptation is aerobic glycolysis or Warburg effect, the ability of cancer cells to enhance glycolysis followed by preferential conversion of pyruvate to lactate, rather than oxidation in the tricarboxylic acid (TCA) cycle, despite the presence of oxygen and functional mitochondria (3). This paradoxical switch to a less efficient way to obtain energy does not disadvantage the cell, provided there is a sufficient nutrient supply, since the increased flux in glycolysis allows it to generate the building blocks and redox power required for anabolic activities. Moreover, these requirements can be satisfied by an increased consumption of alternative substrates such as glutamine and lactate in order to maintain the levels of TCA cycle intermediates $(4,5)$.

In this context, succinate dehydrogenase $(\mathrm{SDH})$ is a critical enzyme complex in the mitochondrial respiratory chain that participates in both the TCA cycle (catalyzing conversion of succinate to fumarate) and oxidative phosphorylation (OXPHOS), where, as a component of complex II, it transfers electrons from succinate to ubiquinone (UbQ) (6). SDH is comprised of four subunits, A through D (SDHA-D), encoded by nuclear DNA. Due to its role in linking two major metabolic pathways, $\mathrm{SDH}$ is tightly regulated and mutations/alterations in member genes that alter complex expression and functionality have been linked to several diseases, including tumor pathologies, such as gastrointestinal tumors, renal cell carcinomas, paragangliomas and phaeochromocytomas (7-9). A deficiency in SDH activity leads to an accumulation of succinate and subsequent hypoxia inducible factor (HIF)-1 $\alpha$ stabilization 
through inhibition of HIF prolyl-hydrolases (PHDs) (10-12). In turn, it is well-established that HIF1 $\alpha$ contributes to tumor progression by enhancing glucose metabolism, promoting angiogenesis and activating oncogenic pathways, such as the mammalian target of rapamycin (mTOR) pathway $(13,14)$. Furthermore, HIF1 $\alpha$ appears to be involved in the modulation of the tumor immunosuppressive phenotype by regulating the immune-checkpoint effectors programmed death-ligand 1 (PD-L1), CD47 and CD73 (15). SDH therefore plays a crucial role interfacing between the tumor metabolome and immune-surveillance pathways (16).

The malignant potential of a tumor is not exclusively dependent on the genetic and epigenetic changes in the cancer cell population but is also influenced by their interaction with other cell types of the tumor microenvironment (TME). This tumor ecosystem is composed of blood vessels, extracellular matrix, soluble signaling molecules, and cells of mesenchymal (fibroblasts, myofibroblasts and mesenchymal stem cells) and hematopoietic (lymphoid and myeloid cells) origin (17). Macrophages constitute one of the most abundant non-tumor cell types in the TME and can display both anti- and pro-tumorigenic features (18). Classically activated macrophages, also called "M1", are involved in the pro-inflammatory (PI) responses of type I T helper $\left(T_{H} 1\right)$ cells to pathogens. They are activated by interferon gamma (IFNY) and toll-like receptor (TLR) signaling and express high levels of major histocompatibility complex II (MHCII), interleukin-12 (IL-12), nitric oxide synthase (NOS) and tumor necrosis factor alpha (TNF $\alpha$ ). By contrast, alternatively activated or "M2" macrophages display anti-inflammatory (Al), type $2 \mathrm{~T}$ helper $\left(\mathrm{T}_{\mathrm{H}} 2\right)$ cell characteristics, such as IL-10 secretion in response to stimulation by IL-4 or IL-13 $(19,20)$. Tumor-associated macrophages (TAMs) exhibit characteristics that are more closely associated with the latter subtype (21), although the complexity and plasticity of the TAM population highlight the need for a more extensive classification (22), with TAMs expressing both PI/M1 and AI/M2 markers (23). TAMs participate in tumor progression by suppressing immune responses and by enhancing tumor cell migration, invasion, extravasation and subsequent metastasis (23). 
An increasing understanding of TAM biology has led to progress in their therapeutic targeting $(24,25)$. The role of activated macrophages in promoting tumor progression can be explained by crosstalk between TAMs and tumor cells. For example, tumor cells release colony stimulating factor 1 (CSF-1) to recruit and educate macrophages, which in turn produce epidermal growth factor (EGF) that contributes to tumor progression (26-28). CSF-1 receptor (CSF-1R) inhibition or ablation reduces tumor progression and metastasis in breast carcinoma and glioma (29-31). In colorectal cancer, TAM-secreted IL-6 enhances cancer cell epithelial to mesenchymal transition (EMT) through phosphorylated signal transducer and activator of transcription 3 (p-STAT3) signaling while TAM-educated tumor cells recruit new TAMs upon chemokine (C-C motif) ligand 2 (CCL2) secretion (32). Other examples include the tumor necrosis factor $\alpha(\mathrm{TNF} \alpha) /$ nuclear factor $\mathrm{KB}(\mathrm{NF}-\mathrm{kB})$ and IL-1 pathways (33-35). Targeting or reprogramming of TAMs towards a pro-inflammatory phenotype (M1) has been demonstrated to inhibit the growth of mammary carcinoma, melanoma and glioblastoma $(34,36,37)$, confirming macrophages as a promising therapeutic target.

In this study, we aimed to understand the role of TAMs in the context of breast cancer metabolism. Our findings suggest that macrophages can affect the expression levels of SDH in tumor cells, therefore having a crucial impact rewiring tumor energetic routes and subsequent cancer progression.

\section{RESULTS}

Macrophages drive changes in metabolism and metabolic gene expression in breast cancer cells.

As reported previously (40-42), breast cancer cells with higher migratory and invasive properties display differential genomic expression profiles, including a metabolic signature or subset of genes with the ability to drive the Warburg effect. Our aim was to elucidate the role of the tumor microenvironment and, more specifically, the role of TAMs in driving this 
metabolic switch within tumor cells. To do this, we used either bone marrow-derived macrophages (BMDM) from mice or peripheral blood mononuclear cells (PBMCs) from human donors (fig. S1A). Differentiation and further polarization of the macrophages was performed ex vivo, resulting in an unpolarized control population (UNP/M0) and two distinct differentiated macrophage populations (Fig. S1, B and C): pro-inflammatory (PI/M1) macrophages exhibited a rounded morphology and secreted pro-inflammatory cytokines, such as IL-12, following stimulation with IFN $\gamma$. In contrast, anti-inflammatory (AI/M2) macrophages were polarized by IL-4 addition and displayed an elongated morphology and a different cytokine secretion profile (increased IL-10 levels), resembling TAMs (43). Accordingly, Al macrophages exhibited diminished expression of the enzyme nitric oxide synthase (NOS2), a marker of proinflammatory polarization, whereas arginase $1(A R G 1)$ was increased in this sub-population (Fig. S1D). Macrophage and breast cancer populations were co-cultured for 24-36 hours, because the secretion of the featured cytokines plateaus after this period (Fig. S1, E and F) and the $\mathrm{PI} / \mathrm{Al}$ classification cannot be confirmed beyond this time point. The macrophage population was then depleted (Fig. S1G), and a metabolite profile characterizing the core energetic routes (Fig. S2A) was obtained from the tumor extracts. Interestingly, principal component analysis showed a distinct metabolic profile for triple-negative breast cancer (TNBC) MDA-MB-231 cells when co-cultured with either PI or Al human PBMC-derived macrophages. Control, unpolarized macrophages also showed a distinct pattern, despite having a wider distribution on the PCA plot compared to the PI/AI co-cultures (Fig. S2B). Individual metabolite concentrations in both $\mathrm{Al}$ and $\mathrm{PI}$ co-cultured cancer cell samples were then compared to the unpolarized population control. Following co-culture with $\mathrm{PI}$ macrophages, the tumor cells exhibited significant higher amounts of glucose in the media suggesting lower glucose utilization, while ATP production was decreased. Moreover, variations in intracellular metabolite levels such as succinate, myo-inositol, alanine or lactate were indicative of a shift towards a less glycolytic phenotype (Fig.1A) in the PI condition (44) with respect to the Al condition. In order to better understand the responsible enzymes for this metabolic switch towards glycolysis, expression of relevant individual genes was performed 
$(45,46)$. While lactate dehydrogenase $(L D H)$ and pyruvate carboxylase $(P C)$ were unaffected

(Fig. S2, C and D), SDH subunit D (SDHD) expression was significantly decreased in two breast cancer cell lines co-cultured with Al macrophages: the aforementioned MDA-MB-231 (human triple negative basal B adenocarcinoma cells) and MCF-7 cells [human estrogen receptor/progesterone receptor-positive, human epidermal growth factor receptor 2-negative (ER+ PR+ HER2-) luminal invasive ductal carcinoma] (Fig. 1, B to D). Decreased SDHD expression in MCF-7 cells was sustained for at least 48 hours following Al macrophage removal (Fig. S2E) suggesting transcriptional regulation driven by the interaction with the macrophage population. Direct interaction between the macrophage and breast cancer cell populations was required, as experiments using paracrine stimulation (transwell) or macrophage-conditioned media did not result in any significant effect on SDH expression levels (Fig. S2F). As previously mentioned, TAMs can share features with both $\mathrm{PI}$ and $\mathrm{Al}$ macrophages and consequently, display intermediate or mixed phenotypes (47). To assess the influence of TAMs in this context, the 4T1 murine TNBC cell line was co-cultured with ex vivo polarized murine $\mathrm{PI} / \mathrm{Al}$ BMDMs or $\left(\mathrm{CD} 11 \mathrm{~b}^{+} \mathrm{F} 4 / 80^{+} \mathrm{Ly} 6 \mathrm{G} / \mathrm{C}^{-}\right)$TAMs extracted from syngeneic 4T1 tumors (Fig. S2G). Notably, TAM co-culture resulted in the highest reduction in SDHD protein levels in 4T1 cells (Fig. 1, E and F) suggesting an in vivo scenario comparable to the in vitro/ex vivo Al co-culture condition. Because of SDH down-regulation, metabolite intracellular concentrations were affected (Fig. 1G). MCF-7 cells co-cultured with Al macrophages exhibited a significantly increased succinate to fumarate ratio, when compared with those co-cultured with PI or control macrophages. As a control, siRNA was used to deplete SDHD in MCF-cells prior to co-culture and in these cells the succinate/fumarate ratio was elevated under all co-culture conditions, and to a level similar to that for control siRNA cells from $\mathrm{Al}$ co-cultures (Fig. $1 \mathrm{G}$ and fig. $\mathrm{S} 2 \mathrm{H}$ ). These findings suggest a direct relation between macrophage subtype and succinate levels in tumor cells. Moreover, they suggest that macrophages have the capacity to drive gene expression changes in neighboring tumor cells, resulting in an alteration of tumor cell metabolism and induction of the Warburg effect. 


\section{STAT and TGF $\beta$ pathways mediate macrophage dependent SDHD expression changes.}

In order to clarify the mechanism underlying the changes in SDH expression, a microarray analysis of murine 4T1 TNBC cells co-cultured with PI or Al murine BMDMs was performed (GEO accession number GSE125457). KEGG database and gene set enrichment data analysis displayed eight pathways in which associated genes were significantly changed when compared between the PI and Al co-cultures ( $p$-value<0.05, FDR<0.3; Data File S1). One of the pathways showing significant differences was the Toll-like receptor (TLR) pathway that includes the transcription factors STAT1 and STAT2 (Fig. 2A). In addition, STAT1 has been described as a binding factor of the $S D H D$ promoter, therefore highlighting it as a valid candidate to regulate SDHD expression (48). Both STAT1 and STAT2 gene expression was significantly upregulated in human MDA-MB-231 and MCF7 breast cancer cells following coculture with PI macrophages (Fig. 2B and fig. S3A). STAT3, a transcription factor from the same family that frequently participates in antagonistic processes (49) exhibited no significant changes in expression (Fig. S3B). The increase in STAT1 mRNA expression observed under PI co-culture conditions was associated with a concomitant increase in STAT1 protein levels (Fig. 2,C and D), while STAT1 phosphorylation status remained proportional to the total level of protein expression (Fig. 2C and fig. S3C). Finally, the level of STAT1 protein bound to the SDHD promoter region of MDA-MB-231 cells was higher in those cells co-cultured with $\mathrm{PI}$ macrophages (Fig. 2E), when compared with those co-cultured with Al macrophages. No differences were observed between the co-cultures for STAT1 binding in an unrelated (ACTB coding for actin) promoter region (Fig. 2F).

We next explored the mechanism of crosstalk between macrophages and tumor cell metabolism. Macrophages secrete a broad array of cytokines that have the capacity to affect signal transduction in neighboring cells. Among them, CD206+ M2-like Al macrophages express high amounts of transforming growth factor beta (TGF $\beta$ ) (50), a major regulator of tumor progression (51). Therefore, we aimed to assess whether disruption of TGF $\beta$ signaling 
would reverse the effects of Al macrophage co-culture on cancer cells. MDA-MB-231 cells were incubated with TGF $\beta$ blocking antibody during co-culture with $\mathrm{PI}$ or Al macrophages. Differences in MDA-MB-231 SDHD protein levels among the control, PI and AI macrophage co-culture groups were abrogated following TGF $\beta$ inhibition (Fig. 2, G and H, and fig. S3D). In addition, STAT1 levels were reduced in the PI co-culture after disruption of TGF $\beta$ signaling, suggesting that the TGF $\beta$ pathway is at least partially involved in the STAT1-dependent transcriptional regulatory mechanisms (Fig. S3, E and F). Collectively, our results suggest that macrophages can control SDH expression through regulation of STAT1 in tumor cells via a mechanism involving TGF $\beta$ signaling.

\section{Al macrophages stabilize HIF1a in tumor cells.}

Succinate has been reported previously to act as an onco-metabolite by inhibiting the PHDs that target HIF1a for proteasomal degradation (10). Therefore, we determined whether macrophages could regulate tumor HIF1a levels by controlling succinate metabolism. Transient siRNA-mediated knockdown of either STAT1 or SDHD resulted in upregulated HIF1 $\alpha$ levels in MCF-7 cells (Fig. S4A). Interaction with Al macrophages increased HIF1 $\alpha$ levels when compared to PI macrophages, in both breast tumor cell lines tested (Figs. 3A and 3B), which is consistent with the observed changes in SDH expression (Fig. 1, C and D) and succinate levels (Fig. 1, A and G). Changes in HIF1a expression occurred in an SDHdependent manner, as these differences are abrogated in SDHD-depleted cells (Fig. 3, C and D, and fig. S4B). Functional HIF1 $\alpha$ levels were assessed using a reporter system where expression of the green fluorescent protein (GFP) is under the control of a HIF1 $1 \alpha$ response element (HRE). MDA-MB-231 cells stably transfected with the HRE>GFP construct displayed GFP expression in response to treatment with $\mathrm{CoCl}_{2}$, which mimics hypoxia, and $\mathrm{O}_{2}$ deprivation (fig. S4, C and D). Time-lapse live cell imaging was then performed to monitor GFP reporter expression in these cells during co-culture with $\mathrm{PI}$ or $\mathrm{Al}$ macrophages labelled with the cell tracker CMTMR (Fig. 3E). Live-cell imaging experiments revealed increased 
HIF1a expression in tumor cells incubated with Al macrophages (Fig. 3, E and F, fig. S4E, and movies S1 and S2). Onset of GFP expression was detected at $\sim 20$ hours post-initiation of co-culture, and at 72 hours, total fluorescence was significantly higher in cells cultured with Al macrophages, when compared with those cultured with PI macrophages (Fig. 3F). In order to assess the role of TGF $\beta$ in macrophage-mediated HIF1 $\alpha$ upregulation, the MDA-MB-231 cell line was co-cultured with macrophages in the presence of anti-TGF $\beta$ blocking antibodies. TGF $\beta$ blockade resulted in a reduction in HIF1 $\alpha$ levels in tumor cells from Al co-cultures (Fig. 3, $\mathrm{G}$ and $\mathrm{H}$ ) and a significant reduction in the number of cells showing GFP activation, when compared with the IgG control (Fig. 3, I and J). Blocking antibodies against IL-10 did not show any effect on HIF1 $\alpha$ stabilization, confirming the specificity of the mechanism of action. These results show that $\mathrm{Al}$ macrophages can trigger HIF1 $\alpha$ upregulation in tumor cells via a TGF $\beta$ dependent mechanism.

Overall, these data suggest a mechanism where TGF $\beta$-mediated interaction between AI macrophages and breast tumor cells triggers gene expression changes in the tumor population, contributing to STAT1-dependent downregulation of SDH expression. This affects basal cancer cell metabolism, by inhibiting TCA cycle activity and favoring a more glycolytic phenotype (the Warburg effect). The accumulation of succinate, resulting from decreased SDH expression, leads to HIF1a stabilization and tumor progression (Fig. S4F).

TAMs regulate tumor metabolism and HIF-dependent vascularization and PD-L1 expression in vivo.

Finally, we aimed to understand whether TAMs could control the metabolism of breast tumor cells in vivo. Tumors arising from the orthotopic implantation of TNBC murine 4T1 cells in BALB/C female mice establish a microenvironment that includes both PI (F4/80+ iNOS+) and Al macrophages (F4/80+ CD163+), as determined by immunofluorescence (Fig. 4A). Using this model, orthotopic tumors were established in mice and endogenous macrophages were 
then depleted with clodronate, an inducer of macrophage apoptosis (Fig S5A). Clodronate administration resulted in a 2 to 3 -fold reduction in the CD11b+ myeloid population in the spleens and tumors of treated mice (Fig. 4B). Quantification of the macrophage subpopulations showed a near complete ablation of the pro-tumorigenic Al macrophages (CD206+ IL4R+, 6-fold decrease) following clodronate administration and a 2-fold reduction in the CD68+ MHCII+ PI population (Fig. S5, B and C). Tumor extracts were profiled using proton-nuclear magnetic resonance $\left({ }^{1} \mathrm{H}-\mathrm{NMR}\right)$. TAM depletion resulted in minor AMP and ATP+ADP production, decreased levels of glycolytic and glutaminolytic metabolites (glycine, alanine, lactate and glutamate) and reduced succinate accumulation (Fig. 4, C to E, and fig. S5D) suggesting a shift from glycolytic to oxidative metabolism. Based on our in vitro findings, we hypothesized that this phenotype could be driven by changes in HIF1 $\alpha$ expression. Histological analysis of tumor sections from clodronate-treated animals showed diminished PD-L1 expression in tumor cells (Fig. 4, F and G) as well as disrupted vasculature formation and reduced expression of the vascular marker CD31 (Fig. 4H), features that are associated directly and indirectly with lower HIF1 $\alpha$ activity $(15,52)$.

Next, we evaluated the ability of ex vivo polarized macrophages to regulate the metabolism of the tumor in clodronate-treated animals. PI/Al macrophages were reintroduced after clodronate treatment and tumors were harvested 48 hours. later (Fig. S6A). Clodronate administration resulted in a 2 to 3 -fold reduction in the $C D 11 b+F 4 / 80+$ macrophage population in the tumors of treated mice (Fig. S6, B and C), numbers that were partially rescued by the ex vivo macrophage reintroduction (Fig. S6C). At the experiment endpoint, $48 \mathrm{~h}$. after macrophage reintroduction, tumor extracts displayed mixed presence of both PI (CD68+ $\mathrm{MHCll}+$ ) and $\mathrm{Al}$ (ILR4+ CD206+) regardless or the injected subtype, suggesting the elevated macrophage plasticity within the tumor environment (Fig. S6D). Nevertheless, Al macrophages showed higher tumor infiltration capacity than their PI counterparts (Figs. S6E and S6F), suggesting differences in the behavior of these macrophage subtypes over the course of the 48-hour period post-injection. Tumor extracts were then profiled using ${ }^{1} \mathrm{H}-\mathrm{NMR}$ 
and sections were analyzed by immunofluorescence. Principal component analysis on metabolomics data showed higher degree of variability within each experimental condition, when compared with the in vitro experiment. Notably, reintroduction of both skewed macrophage populations resulted in a distinct profile when compared with the clodronatetreated or control samples (Fig. S7A). Al macrophage injection caused the highest increase in intra-tumoral succinate and glycolytic substrates such as lactate, alanine or myo-inositol (Figs. 5A, B, C and fig. S7B) thus confirming the ability of ex vivo polarized macrophages to control tumor metabolism and revert it towards the glycolytic type. Immunostaining analysis confirmed that only the exogenous Al macrophages were able to rescue PD-L1 expression in the tumor population (Fig. 5, D and E) and reconstitute CD31 vascular structures (Fig. S7, C and D) when compared with the clodronate-treated only or the reintroduced PI population. Based on these findings, we conclude that TAMs can support immune evasion, angiogenesis and tumor progression through changes in the tumor metabolic patterns.

\section{DISCUSSION}

The tumor microenvironment, and macrophages in particular, serves multiple roles in cancer progression (23) and has become a potential prognostic factor for breast cancer (53). Induction of metabolic changes due to cross-talk between tumor and stroma is currently considered one of the emerging hallmarks of cancer (54). For instance, tumor cells produce lactic acid as a result of aerobic glycolysis that can be taken up by neighboring TAMs, promoting an M2-like polarization in a mechanism that is dependent on Arginase1 (Arg1), VEGF and HIF1 $\alpha$ (55). However, reciprocal mechanisms whereby tumor cell metabolism is regulated by TAMs are poorly understood. Polarized PI macrophages decreased the glycolytic phenotype of tumor cells, measured as lower glucose consumption and ATP production along with decreased glycolysis-derived metabolites such as lactate or myo-inositol. This phenomenon would suggest a metabolic loop between the tumor and macrophage populations in a similar manner to that described for other oncogenic features such as 
migration and metastasis $(27,56)$. This regulation involves changes in expression and activity of the enzyme SDH through the control of the transcription factor STAT1. TGF- $\beta$ is a cytokine typically secreted by Al macrophages that has been reported to downregulate STAT1 signaling in microglia cells (57). Blocking TGF- $\beta$ signaling in vitro in macrophage co-cultures leads to a recovery in SDH expression in tumor cells cultured with Al macrophages. The mechanism of action remains to be fully elucidated and is possibly tumor-type dependent. TGF $\beta$ can abrogate STAT1-dependent transcription (58) suggesting both transcriptional and post-translational regulation. Additionally, released STAT1 transcriptional activity following TFG $\beta$ inhibition may enhance its own transcription via a positive feed-back loop (59).

Downregulation of SDH expression in breast cancer cells resulted in an accumulation of succinate. Succinate has been reported to contribute to tumorigenesis in several ways; promoting angiogenesis through ERK activation (60), inducing epigenetic changes by inhibiting histone demethylases (61), and by stabilizing the transcription factor HIF1 $\alpha$ through either direct inhibition of PHDs (10) or indirectly through increased ROS production (62). We demonstrate that co-culture with PI macrophages triggers a decrease in HIF1 $\alpha$ levels in breast cancer cells. Overexpression of HIF $1 \alpha$ is a characteristic of various types of cancer and has the potential to promote tumorigenic processes, including resistance to apoptosis, invasion and metastasis, adaptation to hypoxia and metabolic changes that support tumor survival and growth (63). HIF1 $\alpha$ drives transcriptional upregulation of nearly all the glycolytic enzymes, including LDH, which catalyzes the conversion of pyruvate to lactate (64). Our results suggest that macrophages have the ability to trigger a switch from oxidative metabolism to aerobic glycolysis based on 1) a TGF- $\beta$ and STAT1-dependent SDH and subsequent TCA cycle regulation and, 2) the stabilization of HIF1 $\alpha$ resulting from increased succinate levels and the corresponding increase in glycolysis.

The macrophage population within the tumor microenvironment is highly variable. In breast tumors, macrophages can account for up to $50 \%$ of the tumor mass (65). In the $4 T 1$ in vivo 
model used in the present study, the macrophage population was substantially smaller and oscillated at $\sim 3 \%$ of the total tumor mass. As expected, it displayed a wide variety of marker expression, with a prevalence of $\mathrm{PI}$ macrophages (CD68+ $\mathrm{MHCll}+$ and $\mathrm{NOS}+)$ relative to $\mathrm{Al}$ macrophages (IL4R+ CD206+ and CD163+). Clodronate liposomes are an efficient tool to deplete macrophages in vivo $(66,67)$. In the $4 \mathrm{~T} 1$ model, clodronate diminished TAMs to less than $1 \%$ of the whole population following treatment. In the absence of TAMs, 4 T1 tumors demonstrated decreased metabolic activity, evidenced by a reduction of glycolytic metabolites (lactate, alanine and glycine), glutaminolytic metabolites (glutamate) and TCA intermediaries (succinate). In vivo succinate accumulation facilitates the stabilization of the oncogenic transcription factor $\operatorname{HIF} 1 \alpha(39,68)$. In addition, the increase in lactate levels acts synergistically to this effect $(69,70)$. Various factors that promote tumorigenesis by enhancing angiogenesis, including VEGF, PDGF and angiopoietins (71), are regulated by HIF1 $\alpha$ (72). Consequently, lack of HIF1 $\alpha$ expression in tumor cells may in part explain the disruption in vasculature formation (indicated by CD31+ vascular structures) upon clodronate treatment (73) and a significant increase in tumor cell death. This phenotype suggests that macrophage ablation in the tumor microenvironment modified the tumor metabolism by reducing its glycolytic activity, resulting in reduced HIF1 $\alpha$-driven angiogenesis. In contrast, reintroduction of $\mathrm{Al}$-skewed macrophages is able to revert this phenotype and reconstitute the vascular structures, thereby showing a direct correlation between macrophage presence and HIF-dependent processes.

HIF1 $\alpha$ regulates the expression of immune checkpoint proteins (15), which are highly expressed in TNBC and correlate with poor patient outcome $(74,75)$. STAT1 also exerts a transcriptional control over PD-L1 (76) and the cytokines IL-10 and TGF $\beta$ contribute to the prevention of anti-tumor immunity (77), overall evidencing a role for Al macrophages in immune evasion in TNBC. Clodronate treatment effectively reduced PD-L1 expression in tumor cells which was rescued with the re-introduction of $\mathrm{Al}$ polarized macrophages. Therefore, ex vivo skewed macrophages show the ability to be recruited to the tumor site and 
exert control over tumor metabolism, vascularization and immune evasion and can constitute an effective therapeutic tool $(78-80)$. However, this effect is limited by the tumor's ability to reeducate macrophages, proving the need to further explore the potential of macrophage targeting as part of an effective combined immunotherapy (80).

In conclusion, pro-tumorigenic macrophages contribute to cancer progression by affecting the central glucose metabolism, angiogenesis and immune evasion within the tumor and their presence may partially explain the limited efficacy of anti-glycolytic treatments $(81,82)$. A novel combined approach should be evaluated to improve the outcome of triple negative breast cancer therapies. For example, the use of nanoparticles to deplete/re-educate the M2like macrophage population within the tumor presents an intriguing therapeutic possibility, given their established association with poor prognosis $(83,84)$.

\section{MATERIALS AND METHODS}

In vivo experiments. $4 \mathrm{~T} 1$ cells $\left(0.25 \times 10^{6}\right)$ were injected subcutaneously into the mammary fat pad of 6-week old BALB/c female mice (Charles River Laboratories). At day 7 after injection, $200 \mu \mathrm{l}$ of phosphate-buffered saline (PBS) or clodronate-containing liposomes (Liposoma) were administered intraperitoneally (3-5 animals/group) every 3 days. At day 12 after injection, (i) tumors were removed aseptically and snap-frozen for metabolomic assays or processed fresh for flow cytometry (fig. 4) or (ii) $1 \times 10^{6}$ ex vivo polarized macrophages were intravenously injected and tumors were processed $48 \mathrm{~h}$. later as described (fig. 5). All animals were maintained under specific pathogen-free conditions and handled in accordance with the Institutional Committees on Animal Welfare of the UK Home Office (The Home Office Animals Scientific Procedures Act, 1986). All animal experiments were approved by the Animal Welfare and Ethical Review Body (AWERB) at University College London (UCL) and carried out under license from the Home Office, UK. 
Cell culture, chemicals, treatments and transfections. MDA-MB-231, MCF-7 and 4T1 cells were maintained in DMEM supplemented with $10 \%$ fetal bovine serum (FBS). Human macrophage monocultures were maintained in IMDM media with 10\% FBS. Co-cultures were maintained using the culture conditions corresponding to the tumor cell line. All media reagents were purchased from Gibco. Exponentially growing cells were plated to achieve consistent confluency (80-90\%) and siRNAs (Ambion; siRNA IDs: SDHD - s12659, STAT1 s279) were then transfected the next day (final concentration: 10nM) using Lipofectamine RNAiMax (Invitrogen) according to the manufacturer's instructions. Control IgG1 and blocking TGF $\beta$ and IL-10 antibodies (BioTechne, $5 \mu \mathrm{g} / \mathrm{ml}$ ) were included in the medium for the duration of the co-culture experiment.

Plasmids and generation of stable cell lines. $p P$ uro. $H R E>G F P$ was generated using the lentiviral expression plasmid PURO.Cre (Addgene plasmid \#17408) as a backbone. Eight repeats of the hypoxia-responsive element (5'-GCCCTACGTGCTGTCTCACACAGC-3') from the 3' enhancer region of the human EPO gene were amplified by PCR, fused to a TATA box/linker (5'-TCTAGAGGGGTATATAATGGAAGCTC-'3) and amplified with flanking restriction enzyme sites ( $5^{\prime}$ Xhol/3' Smal). The constructed fragment, termed HRE, was inserted into PURO.Cre replacing the original PGK promoter. Subsequently, EGFP was amplified by PCR from pLNT/SFFV hNIS-GFP (85) with flanking Smal/Kpnl sites and subcloned into the intermediate construct replacing Cre, thereby providing the final construct pPuro.HRE>GFP. Puromycin (Gibco, $1 \mu \mathrm{g} / \mathrm{ml}$ ) was used as the selection antibiotic.

Murine BMDM isolation and polarization. A bone marrow cell suspension was obtained by flushing out the femurs and tibias of female BALB/C mice. Cells were washed twice with PBS, resuspended in Red Blood Cell Lysing Buffer Hybri-Max (5 min, RT; Sigma), washed twice with PBS and seeded in P100 uncoated petri dishes. Macrophages were differentiated for 7 days by adding recombinant M-CSF (100 ng/ml) and polarized into M1 (10 ng/ml M-CSF, 100 
$\mathrm{ng} / \mathrm{ml}$ IFN $\gamma$ ) or M2 (10 ng/ml M-CSF, $20 \mathrm{ng} / \mathrm{ml} \mathrm{IL-4)} \mathrm{for} 24 \mathrm{~h}$. All recombinant cytokines were from Peprotech.

Human PBMC isolation and macrophage polarization. Human PBMCs were obtained from healthy donors by density gradient centrifugation. Briefly, blood was diluted 2-4 times in cold PBS supplemented with 2mM EDTA and layered on top of Ficoll-Paque (GE Healthcare) at 3:1 ratio. The mix was centrifuged $(400 \times \mathrm{g}, 40 \mathrm{~min}, \mathrm{RT})$ in a swinging-bucket rotor without brake. The mononuclear cell layer ring was carefully transferred to a new tube and washed repeatedly in PBS-EDTA. After pellet resuspension, cells were labelled using a human Pan Monocyte Isolation Kit (Miltenyi Biotec) according to manufacturer's instructions, and then applied to an LS selection column attached to MidiMACS separator (Miltenyi Biotec). The eluted fraction was collected from the column and the cells then seeded. Macrophages were differentiated for 7 days by adding recombinant hGM-CSF $(50 \mathrm{ng} / \mathrm{ml})$ and hM-CSF $(100 \mathrm{ng} / \mathrm{ml})$ to the media to promote $\mathrm{M} 1$ and M2 polarization, respectively. At day 6 post-seeding, M1 cultures were then supplemented with $100 \mathrm{ng} / \mathrm{ml}$ hIFN $\gamma$, while at day 5 post-seeding, M2 cultures were supplemented with $20 \mathrm{ng} / \mathrm{ml}$ hIL-4. All recombinant human cytokines were from Peprotech. Conditioned media was collected from the last $24 \mathrm{~h}$ of macrophage culture and diluted 1:1 with fresh media before addition to cancer cell cultures.

Cell culture separation. Co-cultures of tumor cells and macrophages were harvested by scraping and labelled with magnetic CD11b Microbeads (Miltenyi Biotec), according to manufacturer's instructions. Cell suspension was applied to an LD depletion column attached to MidiMACS separator (Miltenyi Biotec). Tumor cells (unlabeled fraction) were collected after passing through the column and cell purity was then analyzed by flow cytometry using a FITCconjugated anti-CD11b antibody (Miltenyi Biotec). Flow cytometry was performed using an LSRFortessa X-20 flow cytometer (BD Biosciences). 
ELISA. Mouse and human IL1-10 and IL-12 were quantified using the appropriate Mini ELISA Development Kit (Peprotech) according to manufacturer's instructions. Plates were read at $405 \mathrm{~nm}$ with wavelength correction set at $650 \mathrm{~nm}$ using a Varioskan LUX Multimode Microplate Reader (ThermoFisher).

Gene expression analysis. RNA was extracted using RNeasy Mini Kit (Qiagen) according to manufacturer's protocol. RNA concentration and integrity were measured using NanoDrop 1000 spectrophotometer (Thermo Scientific) or 2100 Bioanalyser (Agilent), for RTqPCR and microarray analysis respectively. A total of 500 ng RNA was transcribed using SuperScript III Reverse Transcriptase, according to manufacturer's instructions. For RT-qPCR, the resulting cDNA was used as template for qPCR and amplified with the Power SYBR Green PCR Master Mix (Life Technologies). Three independent experiments each with triplicate reactions were performed and analyzed using a 7500 Real Time PCR System (Applied Biosciences). Relative quantification of the expression levels was determined using the $\Delta \Delta \mathrm{Ct}$ method and normalized to $G A P D H, A C T B$ or $S 6$ transcript amplification. For the microarrays, the resulting cDNA was hybridized in a GCS3000 microarray system (Affymetrix) and the generated data then subjected to Gene Set Enrichment Analysis (GSEA) using the KEGG Pathway Database.

Metabolic assessment of cells and tumors. Metabolic assessment of MDA-MB-231 cells ( $1 \times 10^{7}$ cells per sample) and 4T1 tumors ( 100mg per sample) was performed by NMR and dual phase methanol/water/chloroform extraction as previously described (86). After phase separation, the aqueous fraction was freeze-dried and stored at -80C prior to NMR acquisition. Freeze-dried aqueous extracts were re-dissolved in $600 \mu \mathrm{L}$ deuterated water $\left(D_{2} \mathrm{O}\right.$ containing

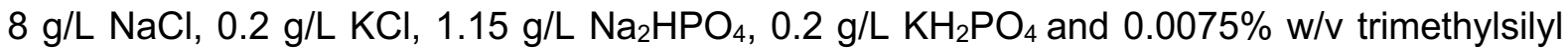
propanoic acid (TSP) as an internal 1H NMR standard for quantification and chemical shift reference). The $\mathrm{pH}$ was adjusted to 7 using $100 \mathrm{mM} \mathrm{HCl}$ or $100 \mathrm{mM} \mathrm{NaOH}$ where necessary. ${ }^{1} \mathrm{H}-\mathrm{NMR}$ spectra were acquired using a NOESY 1D pulse sequence. Cell samples were acquired using a Bruker $500 \mathrm{MHz}$ spectrometer with 512 scans, four dummy scans and 12 
ppm sweep width with 64,000 data points, a repetition time of $7.7 \mathrm{~s}$ per scan, $90^{\circ}$ flip angle and an experiment duration of $67 \mathrm{~min}$. Tumor tissue samples were acquired using a Bruker 4400 $\mathrm{MHz}$ spectrometer with 256 scans, two dummy scans and $14.97 \mathrm{ppm}$ sweep width with 32,000 data points, a repetition time of $5.7 \mathrm{~s}$ per scan, $90^{\circ}$ flip angle and experiment duration of 25 min. TopSpin (version 3.5) software was used for data acquisition and for metabolite quantification. Assignment of metabolites to their respective peaks was carried out based on previously obtained data, confirmed by chemical shift, with reference to published data and using the Chenomx reference database. Peak areas were normalized to the TSP peak and metabolite concentrations quantified per gram of tissue (wet weight) or per million cells. Mean cell metabolite concentrations (fold change) were then calculated. The propagated standard error (SEM) of the ratio was calculated using the formula $S E_{(T / C)}=(T /$ C) $\sqrt{\left(S E_{T} / T\right)^{2}+\left(S E_{C} / C\right)^{2}}$, assuming the covariance between the two groups is zero, i.e. $C$ and T are uncorrelated.

Metabolite quantification. Succinate and fumarate were quantified using a Succinate (Succinic Acid) and Fumarate Colorimetric Assay Kit (BioVision) respectively, according to the manufacturer's instructions. A total of $1 \times 10^{6}$ cancer cells were used per assay and plates were read at $450 \mathrm{~nm}$ wavelength.

Immunoblotting. Cells were washed with ice-cold PBS, harvested by scraping and then homogenized in lysis buffer (CelLytic M, Sigma) supplemented with phosphatase and proteinase inhibitors (PhosSTOP and cOmplete, Roche). Lysates were incubated $\left(1 \mathrm{~h}, 4^{\circ} \mathrm{C}\right)$ and then centrifuged at $14,000 \times \mathrm{g}\left(10 \mathrm{~min}, 4^{\circ} \mathrm{C}\right)$. Protein samples were quantified using the Pierce BCA Protein Assay (ThermoFisher), resolved by SDS-PAGE and transferred to PVDF membranes (Millipore). Membranes were blocked with TBST (50 mM Tris, $150 \mathrm{mM} \mathrm{NaCl}$, $0.5 \%$ Tween $20, \mathrm{pH}=7.5$ ) containing $5 \%$ skim milk powder and probed overnight with primary antibody against SDHD (Millipore), pSTAT and STAT1 (Cell Signaling), GFP (Abcam) or 
HIF1a (BD Biosciences). Bound antibodies were detected by horseradish peroxidase-linked secondary antibodies and processed with Pierce ECL Western Blotting Substrate (ThermoFisher).

Live cell Imaging. Macrophages grown in 6-well plates were incubated with $10 \mu \mathrm{M}$ CellTracker Orange CMTMR Dye (ThermoFisher) for $30 \mathrm{~min}$ at $37^{\circ} \mathrm{C}$ before the addition of MDA-MB-231-HRE>GFP cells to establish co-cultures. Live cell images were acquired every hour over a 72h period using a Nikon Biostation CT Cell Culture Observation System (Nikon). Fluorescence quantification and analysis were performed using Imaris software (Bitplane).

Immunostaining. Tissue sections were fixed in methanol: acetone (1:1) for 20 min at $-20^{\circ} \mathrm{C}$, washed, and then blocked with $2 \%$ BSA $/ 1 \%$ goat serum/TBS for $1 \mathrm{~h}$ at RT. Sections were then incubated with PD-L1 (ThermoFisher), CD31 (Novus Biologicals), HIF1 $\alpha$, F4/80, CD163 and iNOS (Abcam) targeting primary antibodies in blocking buffer overnight at $4^{\circ} \mathrm{C}$ in a humidified chamber. Hoechst 33342 (Life Technologies) was applied for 30 min at RT. Images were acquired using a Zeiss LSM880 with Airyscan confocal microscope.

Flow cytometry. Spleen samples from in vivo experiments were mashed, filtered through a 40- $\mu \mathrm{m}$ cell strainer, incubated in erythrocyte lysis buffer for 3 min at RT, filtered again and finally resuspended in PBS. Tumors were cut into small pieces and resuspended in culture medium containing $1 \mathrm{mg} / \mathrm{ml}$ collagenase type 2 (Worthington Biochemical) and $0.1 \mathrm{mg} / \mathrm{ml}$ DNase I (Boehringer Mannheim), incubated at $37^{\circ} \mathrm{C}$ for $1 \mathrm{~h}$ and then filtered through a $40 \mu \mathrm{m}$ mesh. Samples were centrifuged, washed twice with PBS and stained for 30 min at RT. A list of antibodies used is detailed in Table S1. For co-culture separation confirmation, cell suspensions were stained with a CD11b-FITC marker in order to identify the macrophage subpopulation. Gate settings, including those used for the gating of live single cells in an FSCA versus $\mathrm{FSC}-\mathrm{H}$ plot, were designed based on FMO controls and anti-rat/hamster 
compensation beads (BD Bioscience). TAMs were obtained using a FACSAria cell sorter (BD Biosciences). Data were acquired on a FortessaX20 or a FACSymphony flow cytometer (BD Biosciences) and analyzed with FlowJo (TreeStar).

Chromatin IP. MDA-MB-231 cells were cross-linked with formaldehyde, lysed and sonicated using a Diagenode Bioruptor for 6 x 30s pulses. The resulting whole-cell extract was incubated overnight at $4^{\circ} \mathrm{C}$ with Dynal Protein $\mathrm{G}$ beads (Invitrogen) pre-incubated with $10 \mu \mathrm{g}$ of STAT1 or IgG1 control antibodies (Cell Signaling). Beads were washed, bound complexes were eluted, and crosslinks were reversed by heating at $65^{\circ} \mathrm{C}$. IP and input DNA were then purified by treatment with RNAseA, proteinase $\mathrm{K}$ and phenol: chloroform extraction. Enrichment of specific gene sequences was measured by RTqPCR (Applied Biosystems).

Statistical analysis. Principal Components Analysis was performed using the command prcomp from the $\mathrm{R}$ stats package (87) and plots were produced with the ggbiplot package (88). Data were z-normalized and missing values were inputted with the mean of all valid values for that metabolite. Colocalization and fluorescence quantification analysis were performed on ImageJ using Manders Analysis and Costes automatic threshold (89-91). Five images were acquired from each mouse with three mice per condition. PD-L1 image stacks were collapsed to $2 \mathrm{D}$ using the max intensity projection, and the black level was subtracted from all pixels. All pixels less than grey level 1000 were removed to mask out non-specific signal. The mean of the remaining pixels was calculated to represent each image. CD31 images were quantified by manually counting macroscopic vascular structures per field of view. Graphics and statistical analyses for all figures were performed using GraphPad Prism software. All results represent the mean \pm standard error of the mean, unless stated otherwise. The significance of differences between the means or the population distributions was determined using Two-Way ANOVA test (for metabolomics, live imaging analysis and PD-L1 IF quantification), two-tailed unpaired Student's t-test (for RTqPCR, WB, ELISA and 
colorimetric assays) or non-parametric Mann-Whitney test (for CD31 IF quantification). For all tests, differences were considered statistically significant if $p$-values were $<0.05,0.01$ and 0.001 (indicated with *, ${ }^{* *}$ and ${ }^{* * *}$, respectively, in the figures).

\section{SUPPLEMENTARY MATERIALS}

Figure S1. Macrophage polarization.

Figure S2. Macrophages drive changes in metabolism and metabolic gene expression in breast cancer cells.

Figure S3. STAT and TGF $\beta$ pathways mediate macrophage dependent SDHD expression changes.

Figure S4. Anti-inflammatory macrophages stabilize HIF1 $\alpha$ levels and transcriptional activity.

Figure S5. Tumor-associated macrophages regulate tumor metabolism in vivo.

Figure S6. Ex vivo polarized macrophages are recruited to the tumor site in an orthotopic model.

Figure S7. Ex vivo polarized macrophages regulate in vivo tumor metabolism, vascularization and immune evasion.

Table S1. List of antibodies used in flow-cytometry experiments for macrophage characterization.

Data File S1. Excel file containing microarray KEGG analysis and individual gene list.

Movie S1. HIF1 $\alpha$ activation in MDA-MB-231 cancer cells co-cultured with PI macrophages..

Movie S2. HIF1 $\alpha$ activation in MDA-MB-231 cancer cells co-cultured with Al macrophages..

\section{REFERENCES AND NOTES}

1. D. Hanahan, R. A. Weinberg, Hallmarks of cancer: the next generation. Cell 144, 646-674 (2011)

2. R. A. Cairns, I. Harris, S. McCracken, T. W. Mak, Cancer cell metabolism. Cold Spring Harb Symp Quant Biol 76, 299-311 (2011). 
3. O. Warburg, On the origin of cancer cells. Science 123, 309-314 (1956).

4. M. G. Vander Heiden, L. C. Cantley, C. B. Thompson, Understanding the Warburg effect: the metabolic requirements of cell proliferation. Science 324, 1029-1033 (2009).

5. P. Danhier et al., Cancer metabolism in space and time: Beyond the Warburg effect. Biochim Biophys Acta 1858, 556-572 (2017).

6. A. Bezawork-Geleta, J. Rohlena, L. Dong, K. Pacak, J. Neuzil, Mitochondrial Complex II: At the Crossroads. Trends Biochem Sci 42, 312-325 (2017).

7. M. Rinelli et al., Pediatric gastrointestinal stromal tumor: Report of two novel patients harboring germline variants in SDHB and SDHC genes. Cancer Genet 241, 61-65 (2020).

8. J. Turchini, A. J. Gill, Morphologic Clues to Succinate Dehydrogenase (SDH) Deficiency in Pheochromocytomas and Paragangliomas. Am J Surg Pathol 44, 422-424 (2020).

9. A. J. Gill et al., Succinate dehydrogenase (SDH)-deficient renal carcinoma: a morphologically distinct entity: a clinicopathologic series of 36 tumors from 27 patients. Am J Surg Pathol 38, 1588-1602 (2014).

10. M. A. Selak et al., Succinate links TCA cycle dysfunction to oncogenesis by inhibiting HIFalpha prolyl hydroxylase. Cancer Cell 7, 77-85 (2005).

11. E. M. Palmieri et al., Pharmacologic or Genetic Targeting of Glutamine Synthetase Skews Macrophages toward an M1-like Phenotype and Inhibits Tumor Metastasis. Cell Rep 20, 1654-1666 (2017).

12. G. Xiong et al., Collagen prolyl 4-hydroxylase 1 is essential for HIF-1alpha stabilization and TNBC chemoresistance. Nat Commun 9, 4456 (2018).

13. G. L. Semenza, Oxygen sensing, homeostasis, and disease. N Engl J Med 365, 537-547 (2011).

14. H. S. Zhang et al., NRF2 facilitates breast cancer cell growth via HIF1a-mediated metabolic reprogramming. Int J Biochem Cell Biol 95, 85-92 (2018).

15. D. Samanta et al., Chemotherapy induces enrichment of CD47(+)/CD73(+)/PDL1(+) immune evasive triple-negative breast cancer cells. Proc Natl Acad Sci U S A 115, E1239-E1248 (2018).

16. R. Ramapriyan et al., Altered cancer metabolism in mechanisms of immunotherapy resistance. Pharmacol Ther, (2018).

17. D. R. Pattabiraman, R. A. Weinberg, Tackling the cancer stem cells - what challenges do they pose? Nat Rev Drug Discov 13, 497-512 (2014).

18. A. Mantovani, S. Sozzani, M. Locati, P. Allavena, A. Sica, Macrophage polarization: tumorassociated macrophages as a paradigm for polarized M2 mononuclear phagocytes. Trends Immunol 23, 549-555 (2002).

19. M. C. Schmid, J. A. Varner, Myeloid cells in the tumor microenvironment: modulation of tumor angiogenesis and tumor inflammation. J Oncol 2010, 201026 (2010).

20. D. G. DeNardo et al., CD4(+) T cells regulate pulmonary metastasis of mammary carcinomas by enhancing protumor properties of macrophages. Cancer Cell 16, 91-102 (2009).

21. A. Mantovani, A. Sica, Macrophages, innate immunity and cancer: balance, tolerance, and diversity. Curr Opin Immunol 22, 231-237 (2010).

22. J. W. Pollard, Trophic macrophages in development and disease. Nat Rev Immuno/ 9, 259270 (2009).

23. B. Z. Qian, J. W. Pollard, Macrophage diversity enhances tumor progression and metastasis. Cell 141, 39-51 (2010).

24. R. Noy, J. W. Pollard, Tumor-associated macrophages: from mechanisms to therapy. Immunity 41, 49-61 (2014).

25. C. B. Williams, E. S. Yeh, A. C. Soloff, Tumor-associated macrophages: unwitting accomplices in breast cancer malignancy. NPJ Breast Cancer 2, (2016).

26. J. Condeelis, J. W. Pollard, Macrophages: obligate partners for tumor cell migration, invasion, and metastasis. Cell 124, 263-266 (2006).

27. J. Wyckoff et al., A paracrine loop between tumor cells and macrophages is required for tumor cell migration in mammary tumors. Cancer Res 64, 7022-7029 (2004). 
28. J. B. Wyckoff et al., Direct visualization of macrophage-assisted tumor cell intravasation in mammary tumors. Cancer Res 67, 2649-2656 (2007).

29. E. Y. Lin, A. V. Nguyen, R. G. Russell, J. W. Pollard, Colony-stimulating factor 1 promotes progression of mammary tumors to malignancy. J Exp Med 193, 727-740 (2001).

30. S. M. Pyonteck et al., CSF-1R inhibition alters macrophage polarization and blocks glioma progression. Nat Med 19, 1264-1272 (2013).

31. A. Patsialou et al., Invasion of human breast cancer cells in vivo requires both paracrine and autocrine loops involving the colony-stimulating factor-1 receptor. Cancer Res 69, 9498-9506 (2009).

32. C. Wei et al., Crosstalk between cancer cells and tumor associated macrophages is required for mesenchymal circulating tumor cell-mediated colorectal cancer metastasis. Mol Cancer 18, 64 (2019).

33. E. Voronov, R. N. Apte, IL-1 in Colon Inflammation, Colon Carcinogenesis and Invasiveness of Colon Cancer. Cancer Microenviron 8, 187-200 (2015).

34. Z. Zhu et al., Yin-yang effect of tumour cells in breast cancer: from mechanism of crosstalk between tumour-associated macrophages and cancer-associated adipocytes. Am J Cancer Res 10, 383-392 (2020).

35. D. Saha, R. L. Martuza, S. D. Rabkin, Macrophage Polarization Contributes to Glioblastoma Eradication by Combination Immunovirotherapy and Immune Checkpoint Blockade. Cancer Cell 32, 253-267 e255 (2017).

36. A. M. Georgoudaki et al., Reprogramming Tumor-Associated Macrophages by Antibody Targeting Inhibits Cancer Progression and Metastasis. Cell Rep 15, 2000-2011 (2016).

37. K. Binnemars-Postma, G. Storm, J. Prakash, Nanomedicine Strategies to Target TumorAssociated Macrophages. Int J Mol Sci 18, (2017).

38. H. J. Lehtonen et al., Increased HIF1 alpha in SDH and FH deficient tumors does not cause microsatellite instability. Int J Cancer 121, 1386-1389 (2007).

39. P. J. Pollard et al., Accumulation of Krebs cycle intermediates and over-expression of HIF1alpha in tumours which result from germline FH and SDH mutations. Hum Mol Genet 14, 2231-2239 (2005).

40. R. Limame et al., Expression profiling of migrated and invaded breast cancer cells predicts early metastatic relapse and reveals Kruppel-like factor 9 as a potential suppressor of invasive growth in breast cancer. Oncoscience 1, 69-81 (2014).

41. A. Patsialou et al., Selective gene-expression profiling of migratory tumor cells in vivo predicts clinical outcome in breast cancer patients. Breast Cancer Res 14, R139 (2012).

42. W. Wang et al., Coordinated regulation of pathways for enhanced cell motility and chemotaxis is conserved in rat and mouse mammary tumors. Cancer Res 67, 3505-3511 (2007).

43. A. Sica, A. Mantovani, Macrophage plasticity and polarization: in vivo veritas. J Clin Invest 122, 787-795 (2012).

44. S. Cassim, V. A. Raymond, B. Lacoste, P. Lapierre, M. Bilodeau, Metabolite profiling identifies a signature of tumorigenicity in hepatocellular carcinoma. Oncotarget 9, 26868-26883 (2018).

45. S. Cardaci et al., Pyruvate carboxylation enables growth of SDH-deficient cells by supporting aspartate biosynthesis. Nat Cell Biol 17, 1317-1326 (2015).

46. C. Lussey-Lepoutre et al., Loss of succinate dehydrogenase activity results in dependency on pyruvate carboxylation for cellular anabolism. Nat Commun 6, 8784 (2015).

47. Y. Lin, J. Xu, H. Lan, Tumor-associated macrophages in tumor metastasis: biological roles and clinical therapeutic applications. J Hematol Oncol 12, 76 (2019).

48. S. P. Pitroda et al., STAT1-dependent expression of energy metabolic pathways links tumour growth and radioresistance to the Warburg effect. BMC Med 7, 68 (2009). 
49. K. Friedrich, H. Dolznig, X. Han, R. Moriggl, Steering of carcinoma progression by the YIN/YANG interaction of STAT1/STAT3. Biosci Trends 11, 1-8 (2017).

50. A. Nawaz et al., CD206(+) M2-like macrophages regulate systemic glucose metabolism by inhibiting proliferation of adipocyte progenitors. Nat Commun 8, 286 (2017).

51. C. J. David, J. Massague, Contextual determinants of TGFbeta action in development, immunity and cancer. Nat Rev Mol Cell Biol, (2018).

52. G. Musumeci et al., Enhanced expression of CD31/platelet endothelial cell adhesion molecule 1 (PECAM1) correlates with hypoxia inducible factor-1 alpha (HIF-1alpha) in human glioblastoma multiforme. Exp Cell Res 339, 407-416 (2015).

53. X. Tang, Tumor-associated macrophages as potential diagnostic and prognostic biomarkers in breast cancer. Cancer Lett 332, 3-10 (2013).

54. N. N. Pavlova, C. B. Thompson, The Emerging Hallmarks of Cancer Metabolism. Cell Metab 23, 27-47 (2016).

55. O. R. Colegio et al., Functional polarization of tumour-associated macrophages by tumourderived lactic acid. Nature 513, 559-563 (2014).

56. S. Su et al., A positive feedback loop between mesenchymal-like cancer cells and macrophages is essential to breast cancer metastasis. Cancer Cell 25, 605-620 (2014).

57. X. Zhou, T. Zoller, K. Krieglstein, B. Spittau, TGFbeta1 inhibits IFNgamma-mediated microglia activation and protects mDA neurons from IFNgamma-driven neurotoxicity. J Neurochem 134, 125-134 (2015).

58. C. Reardon, D. M. McKay, TGF-beta suppresses IFN-gamma-STAT1-dependent gene transcription by enhancing STAT1-PIAS1 interactions in epithelia but not monocytes/macrophages. J Immunol 178, 4284-4295 (2007).

59. K. Yuasa, T. Hijikata, Distal regulatory element of the STAT1 gene potentially mediates positive feedback control of STAT1 expression. Genes Cells 21, 25-40 (2016).

60. X. Mu et al., Oncometabolite succinate promotes angiogenesis by upregulating VEGF expression through GPR91-mediated STAT3 and ERK activation. Oncotarget 8, 13174-13185 (2017).

61. M. Xiao et al., Inhibition of alpha-KG-dependent histone and DNA demethylases by fumarate and succinate that are accumulated in mutations of FH and SDH tumor suppressors. Genes Dev 26, 1326-1338 (2012).

62. T. Ishii et al., A mutation in the SDHC gene of complex II increases oxidative stress, resulting in apoptosis and tumorigenesis. Cancer Res 65, 203-209 (2005).

63. G. L. Semenza, Targeting HIF-1 for cancer therapy. Nat Rev Cancer 3, 721-732 (2003).

64. J. D. Firth, B. L. Ebert, P. J. Ratcliffe, Hypoxic regulation of lactate dehydrogenase A. Interaction between hypoxia-inducible factor 1 and cAMP response elements. J Biol Chem 270, 21021-21027 (1995).

65. E. Obeid, R. Nanda, Y. X. Fu, O. I. Olopade, The role of tumor-associated macrophages in breast cancer progression (review). Int J Oncol 43, 5-12 (2013).

66. G. Galletti et al., Targeting Macrophages Sensitizes Chronic Lymphocytic Leukemia to Apoptosis and Inhibits Disease Progression. Cell Rep 14, 1748-1760 (2016).

67. N. van Rooijen, A. Sanders, Elimination, blocking, and activation of macrophages: three of a kind? J Leukoc Biol 62, 702-709 (1997).

68. P. Koivunen et al., Inhibition of hypoxia-inducible factor (HIF) hydroxylases by citric acid cycle intermediates: possible links between cell metabolism and stabilization of HIF. J Biol Chem 282, 4524-4532 (2007).

69. P. Sonveaux et al., Targeting the lactate transporter MCT1 in endothelial cells inhibits lactate-induced HIF-1 activation and tumor angiogenesis. PLoS One 7, e33418 (2012).

70. L. Iommarini, A. M. Porcelli, G. Gasparre, I. Kurelac, Non-Canonical Mechanisms Regulating Hypoxia-Inducible Factor 1 Alpha in Cancer. Front Oncol 7, 286 (2017). 
71. J. W. Pollard, Tumour-educated macrophages promote tumour progression and metastasis. Nat Rev Cancer 4, 71-78 (2004).

72. B. L. Krock, N. Skuli, M. C. Simon, Hypoxia-induced angiogenesis: good and evil. Genes Cancer 2, 1117-1133 (2011).

73. S. M. Zeisberger et al., Clodronate-liposome-mediated depletion of tumour-associated macrophages: a new and highly effective antiangiogenic therapy approach. Br J Cancer 95, 272-281 (2006).

74. E. A. Mittendorf et al., PD-L1 expression in triple-negative breast cancer. Cancer Immunol Res 2, 361-370 (2014).

75. L. Buisseret et al., Clinical significance of CD73 in triple-negative breast cancer: multiplex analysis of a phase III clinical trial. Ann Oncol 29, 1056-1062 (2018).

76. I. Zerdes, A. Matikas, J. Bergh, G. Z. Rassidakis, T. Foukakis, Genetic, transcriptional and posttranslational regulation of the programmed death protein ligand 1 in cancer: biology and clinical correlations. Oncogene 37, 4639-4661 (2018).

77. D. M. Pardoll, The blockade of immune checkpoints in cancer immunotherapy. Nat Rev Cancer 12, 252-264 (2012).

78. P. F. Ma et al., Cytotherapy with M1-polarized macrophages ameliorates liver fibrosis by modulating immune microenvironment in mice. J Hepatol 67, 770-779 (2017).

79. A. J. Perise-Barrios et al., Use of carbosilane dendrimer to switch macrophage polarization for the acquisition of antitumor functions. Nanoscale 7, 3857-3866 (2015).

80. C. M. Alvey et al., SIRPA-Inhibited, Marrow-Derived Macrophages Engorge, Accumulate, and Differentiate in Antibody-Targeted Regression of Solid Tumors. Curr Bio/ 27, 2065-2077 e2066 (2017).

81. N. Hay, Reprogramming glucose metabolism in cancer: can it be exploited for cancer therapy? Nat Rev Cancer 16, 635-649 (2016).

82. A. Luengo, D. Y. Gui, M. G. Vander Heiden, Targeting Metabolism for Cancer Therapy. Cell Chem Biol 24, 1161-1180 (2017).

83. D. Reichel, M. Tripathi, J. M. Perez, Biological Effects of Nanoparticles on Macrophage Polarization in the Tumor Microenvironment. Nanotheranostics 3, 66-88 (2019).

84. F. Castro et al., Pro-inflammatory chitosan/poly(gamma-glutamic acid) nanoparticles modulate human antigen-presenting cells phenotype and revert their pro-invasive capacity. Acta Biomater 63, 96-109 (2017).

85. A. Volpe et al., Radionuclide-fluorescence Reporter Gene Imaging to Track Tumor Progression in Rodent Tumor Models. J Vis Exp, (2018).

86. Y. L. Chung, M. O. Leach, T. R. Eykyn, Magnetic Resonance Spectroscopy to Study Glycolytic Metabolism During Autophagy. Methods Enzymol 588, 133-153 (2017).

87. R. Core Team, A language and environment for statistical computing. R Foundation for Statistical Computing. (2018).

88. V. Q. Vu, ggbiplot: A ggplot2 based biplot. R package version 0.55. (2011).

89. J. Schindelin et al., Fiji: an open-source platform for biological-image analysis. Nat Methods 9, 676-682 (2012).

90. E. M. Manders, J. Stap, G. J. Brakenhoff, R. van Driel, J. A. Aten, Dynamics of threedimensional replication patterns during the S-phase, analysed by double labelling of DNA and confocal microscopy. J Cell Sci 103 ( Pt 3), 857-862 (1992).

91. S. V. Costes et al., Automatic and quantitative measurement of protein-protein colocalization in live cells. Biophys J 86, 3993-4003 (2004).

ACKNOWLEDGMENTS: The authors thank Professor Margaret Ashcroft at University of Cambridge and Dr. James Monypenny for their intellectual feedback; Dr. Eleni Skourti at 
King's College London, Dr. Matthew Arno at the Genomics Centre, King's College London, Mathew Robson at the CR-UK In Vivo Core Facility and the CR-UK Microscopy and Imaging Core Facility at the Cancer Institute, UCL for providing technical expertise. FUNDING: This work was supported by the CRUK City of London Centre (C7893/A26233), King's College London-UCL Comprehensive Cancer Imaging Centre (CRUK and EPSRC, grants C1519/A10331 and C1519/A16463), in association with the MRC and DoH (grant C1519/A10331) and by KCL Breast Cancer Now Unit funding (for F.F.B.; grant KCL-Q2-Y5). We also acknowledge support from the NIHR Biomedical Research Centre at Guy's and St Thomas' NHS Foundation Trust and King's College London and the Centre of Excellence in Medical Engineering funded by the Wellcome Trust and Engineering and Physical Sciences Research Council (EPSRC) (WT 088641/Z/09/Z). AUTHOR CONTRIBUTIONS: T.N. conceived, supervised, and secured funding for the project. V.G. conceived and conducted experiments and performed data analysis. T.R.E. conducted and analyzed ${ }^{1} \mathrm{H}-\mathrm{NMR}$ experiments. V.M., R.M., F.F.B., R.G., F.P. and C.W.L. conducted experiments. P.B. performed PCA and provided statistical guidance. A.P., G.F., S.R. and K.M.B. provided expertise and intellectual feedback. V.G and T.N. wrote the manuscript. COMPETING INTERESTS: The authors declare that they have no competing interests. DATA AVAILABILITY: All data generated or analyzed during this study are included in the main manuscript and its supplementary files. The microarray data have been deposited in NCBI's Gene Expression Omnibus (GEO) and are accessible through GEO Series accession number GSE125457 (https://www.ncbi.nIm.nih.gov/geo/query/acc.cgi?acc=GSE125457). 


\section{FIGURE LEGENDS}

A

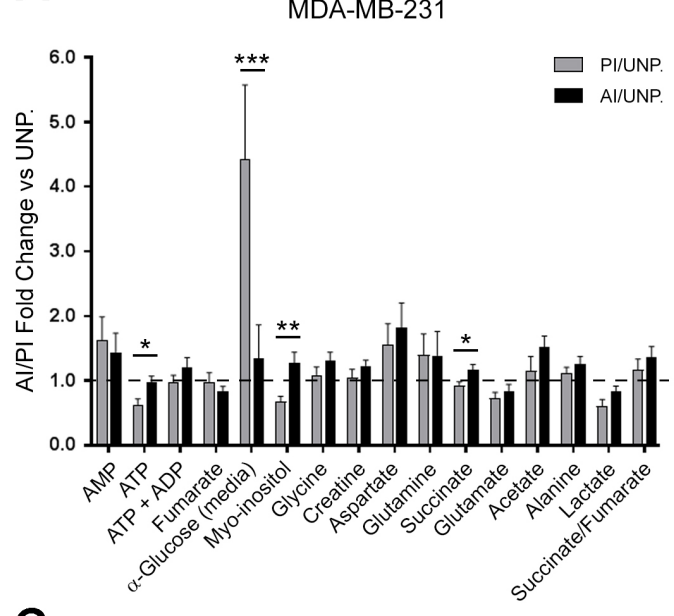

C

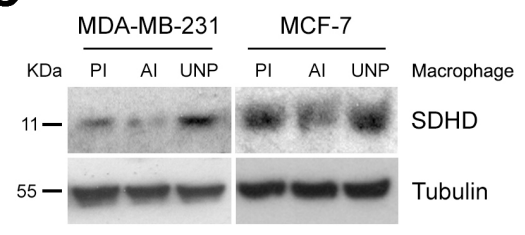

E

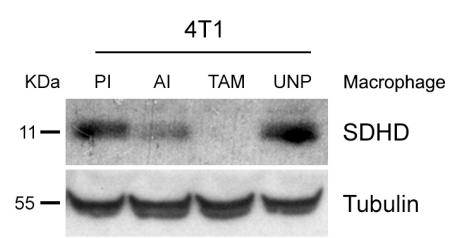

B

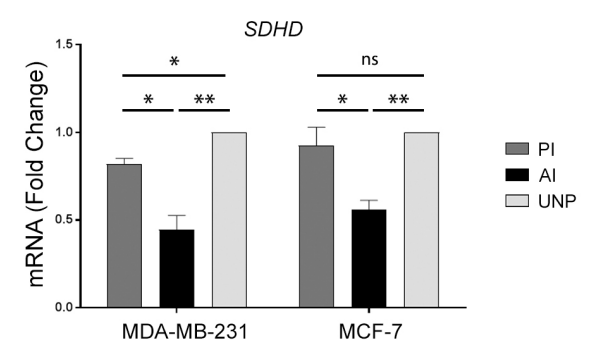

D

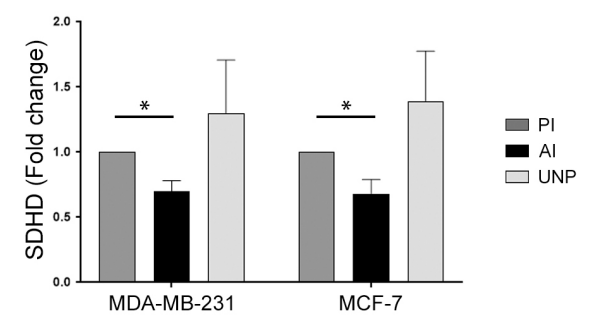

G

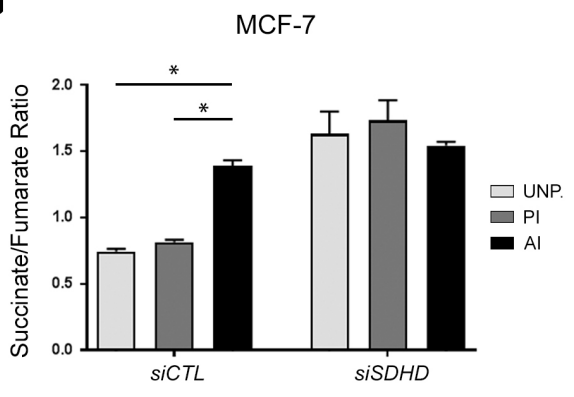

Figure 1. Macrophages promote changes in metabolism and metabolic gene expression in breast cancer cells. (A) Metabolite quantification by ${ }^{1} \mathrm{H}-\mathrm{NMR}$. Results show the metabolite ratio in MDA-MB-231 cells co-cultured with pro- or anti-inflammatory $(\mathrm{PI}, \mathrm{Al})$ macrophages relative to cancer cells co-cultured with control unpolarized (UNP) macrophages. Results are shown as mean \pm SE ( $n=4$ independent replicates for UNP and $n=8$ for PI/Al; two-way ANOVA with $t$-test multiple comparisons, $\left.{ }^{*} P<0.05,{ }^{* *} P<0.01\right)$. (B) Quantitative real-time PCR analysis of $S D H D$ gene expression in MDA-MB-231 and MCF-7 breast cancer cell lines following PI, Al or UNP macrophage co-culture. Results are normalized to the UNP macrophage co-culture and shown as mean \pm SE ( $n=3$ independent experiments; Student's $t$-test, ${ }^{*} P<0.05 ;{ }^{*} P<0.01$; 
and ns, not significant). (C and D) Western blot analysis of SDHD expression in MDA-MB-231 and MCF-7 breast cancer cells following PI, Al or UNP macrophage co-culture. Results show one representative experiment and quantifications are normalized to the PI macrophage coculture and expressed as mean $\pm S E(n=3$ independent experiments; * $P<0.05$ by Student's ttest). ( $E$ and F) Western blot analysis of SDHD expression in 4T1 murine breast cancer cells after co-culture with UNP, PI, AI or TAM macrophages. Results show one representative experiment and quantifications are expressed as mean $\pm S D(n=2$ independent experiments; one-way ANOVA with Welch's $t$-test multiple comparisons, $\left.{ }^{* *} P<0.001\right)$. (G) Metabolite quantification in MCF-7 cells co-cultured with UNP, PI or AI macrophages and transfected with the indicated siRNAs. The succinate-to-fumarate ratio is shown as mean \pm SE $(n=3$ independent experiments; Student's $t$-test, $\left.{ }^{*} P<0.05\right)$. 
A

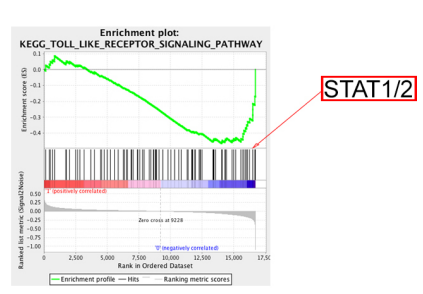

C

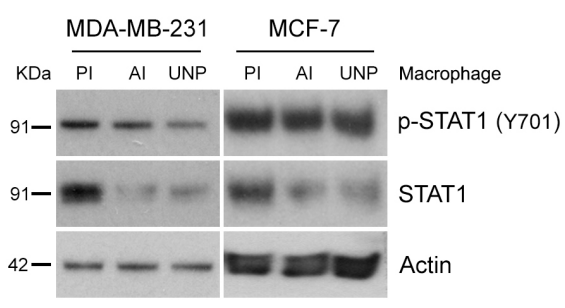

E

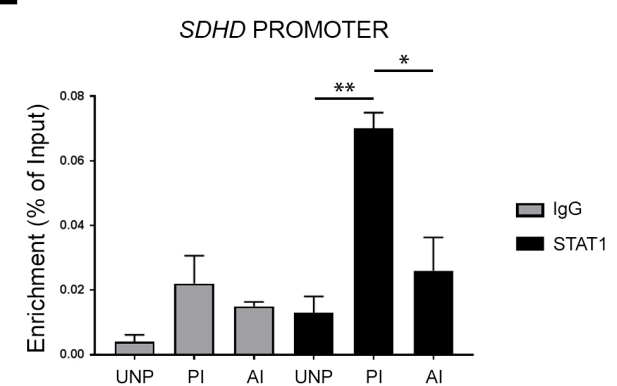

G

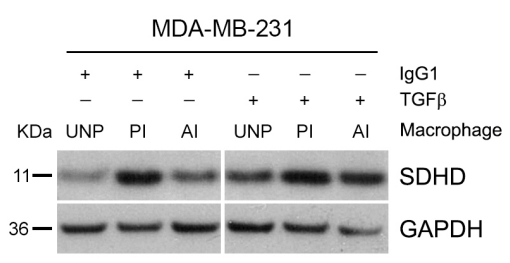

B

STAT1

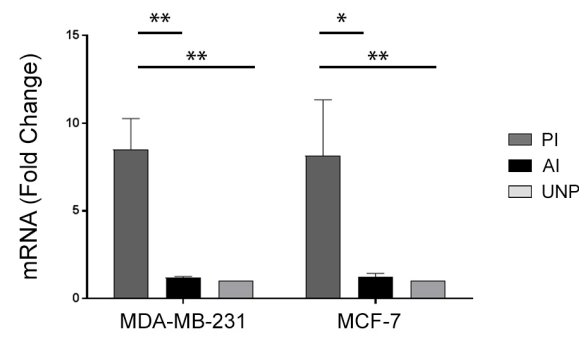

D

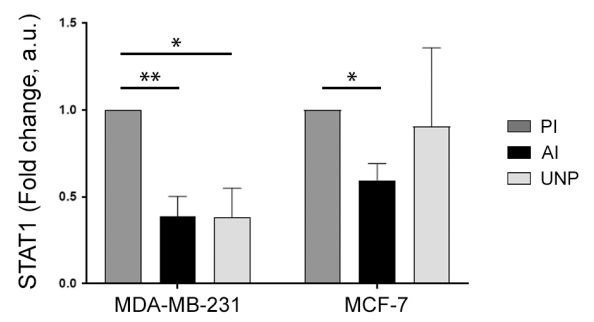

$\mathbf{F}$
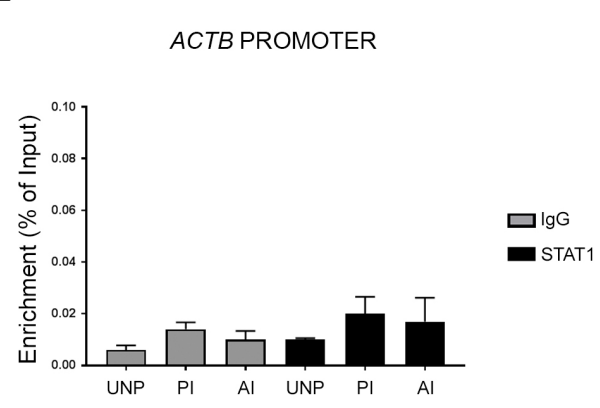

H

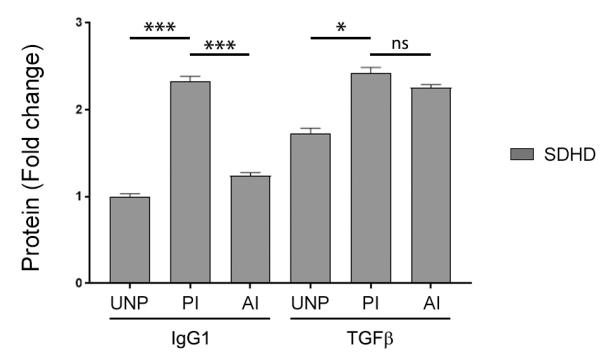

Figure 2. STAT and TGF $\beta$ pathways mediate macrophage-dependent SDHD expression changes. (A) Gene Set Enrichment Analysis (GSEA) algorithm for the KEGG Pathway Database reveals a negative enrichment of the Toll-like receptor signaling pathway in 4T1 murine breast cancer cells following anti-inflammatory (AI) macrophage co-culture, normalized to the pro-inflammatory (PI) co-culture experimental group. The STAT1/2 transcription factors are highlighted. (B) Quantitative real-time PCR analysis of STAT1 gene expression in MDAMB-231 and MCF-7 breast cancer cells following UNP, PI or Al macrophage co-culture. 
Results are normalized to the UNP macrophage co-culture and shown as mean \pm SE $(n=3$ independent experiments; Student's $t$-test, ${ }^{*} P<0.05$, $\left.{ }^{* *} P<0.01\right)$. (C and D) Western blotting for STAT1 phosphorylation (at $\mathrm{Tyr}^{701}$ ) and total STAT1 abundance in MDA-MB-231 and MCF7 breast cancer cells following UNP, PI or Al macrophage co-culture. Results show one representative experiment and quantifications are normalized to the PI macrophage co-culture and expressed as mean \pm SE ( $n=3$ independent experiments; Student's $t$-test, ${ }^{*} P<0.05$, $\left.{ }^{* *} P<0.01\right)$. (E and F) Chromatin immunoprecipitation followed by quantitative qPCR analysis of the $S D H D$ promoter $(\mathrm{E})$ or the $A C T B(\mathrm{~F})$ promoter in MDA-MB-231 cells after co-culture with UNP, PI and Al macrophages. Cross-linked DNA-protein samples were pulled-down using STAT1 (black bars) or control (grey bars) antibodies. Results are expressed as percentage of enrichment compared with the whole cell lysate (input) and shown as mean \pm SD ( $\mathrm{n}=3$; independent experiments; Student's $t$-test, ${ }^{*} P<0.05$, $\left.{ }^{* *} P<0.01\right)$. (G and H) Western Blot showing SDHD expression in MDA-MB-231 breast cancer cells after UNP, PI, or AI macrophage co-cultures were treated with IgG1 control or TGF $\beta$-blocking antibodies. Results show one representative experiment and quantifications are normalized to the UNP CTL macrophage co-culture and expressed as mean \pm SE ( $n=3$ independent experiments; Student's $t$-test, ${ }^{*} P<0.05 ;{ }^{* * *} P<0.001$; and ns, not significant). 


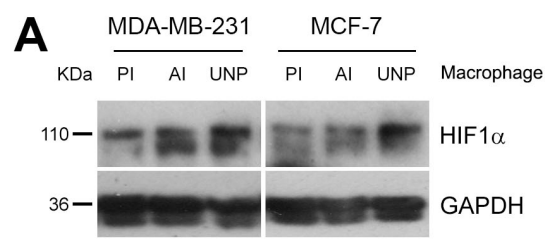

B

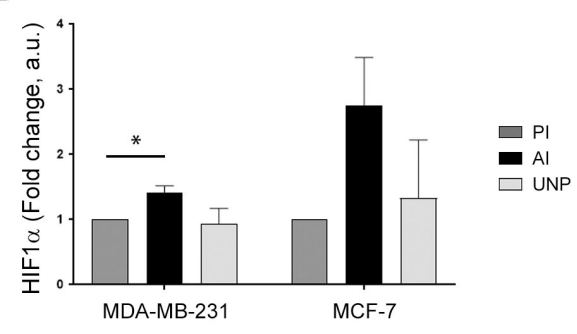

C

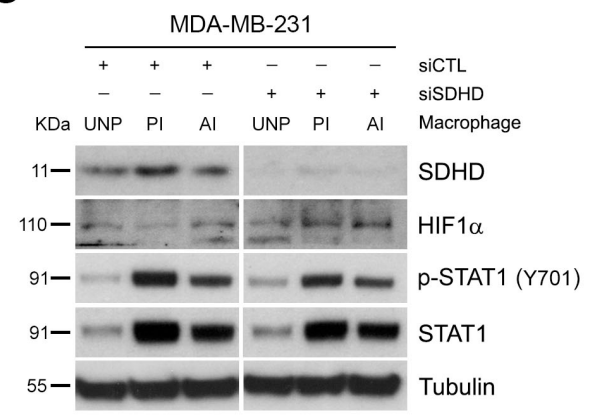

D

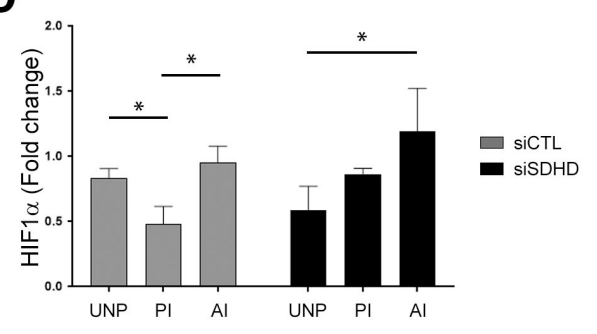

$\mathbf{E}$

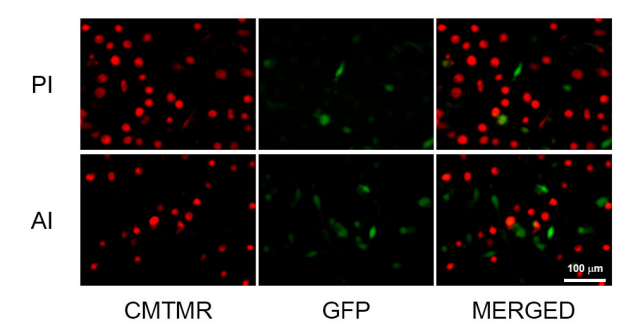

$\mathbf{F}$

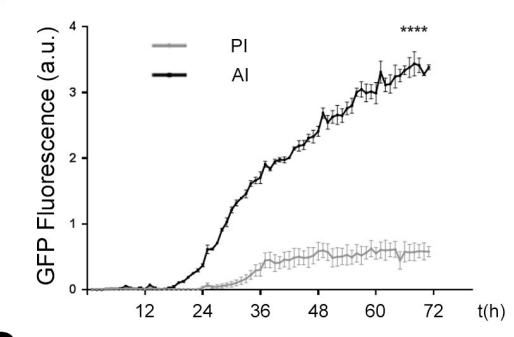

G

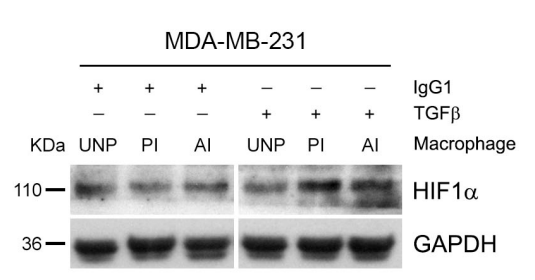

H

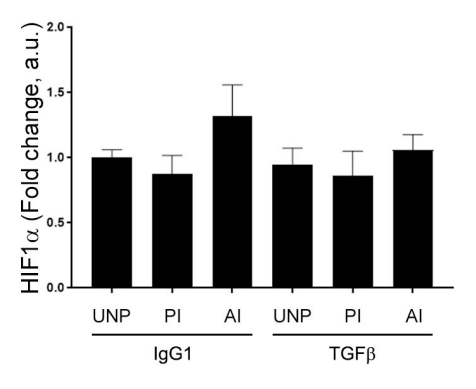

I

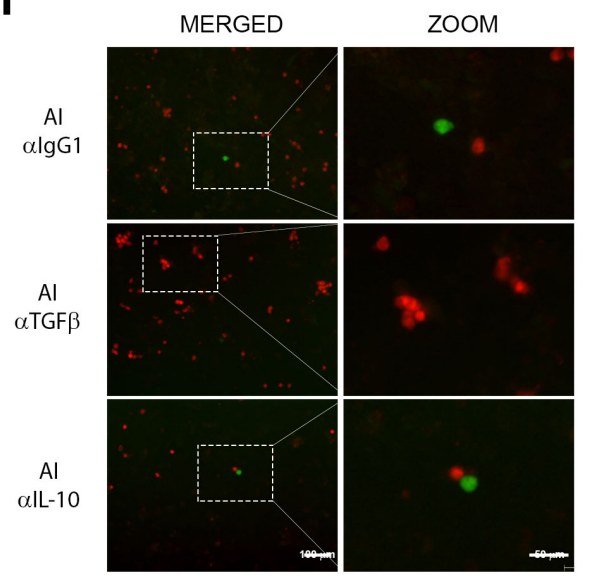

$\mathbf{J}$

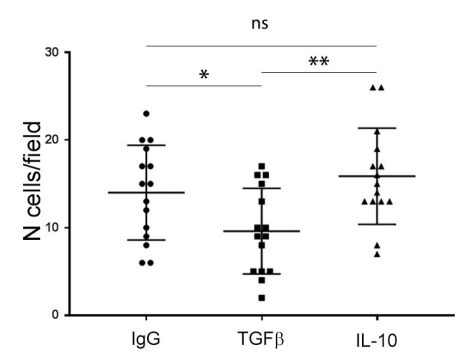

Figure 3. Anti-inflammatory macrophages stabilize HIF1 $\alpha$ levels and transcriptional activity. (A and B) Western blot showing HIF1 $\alpha$ expression in MDA-MB-231 and MCF-7 
breast cancer cells following unpolarized (UNP), proinflammatory (PI) or anti-inflammatory (Al) macrophage co-culture. Results show one representative experiment and quantifications are normalized to the PI macrophage co-culture and expressed as mean \pm SE $(n=3$ independent experiments; Student's I-test, ${ }^{*} P<0.05$ ). (C and D) Western blot showing expression of the indicated proteins in control or SDHD-depleted MDA-MB-231 breast cancer cells following UNP, PI or Al macrophage co-culture. Results show one representative experiment and quantifications are expressed as mean \pm SE ( $n=3$ independent experiments; Student's I-test, $\left.{ }^{*} P<0.05\right)$. (E and F) Representative microscopy images showing the labelling of macrophages (CMTMR cell tracker) and breast cancer cells (GFP) in a co-culture of MDA-MB-231HRE>GFP cells with PI or AI macrophages. Live-cell images were acquired every hour for 72 hours. Scale bar, $50 \mu \mathrm{m}$. Quantification of GFP fluorescence are shown as mean \pm SE $(n=5$ independent experiments; two-way ANOVA, ${ }^{* * * *} P<0.0001$ ). ( $\mathbf{G}$ and $\mathbf{H}$ ) Western blotting for HIF1 $\alpha$ expression in MDA-MB-231 breast cancer cells from UNP, PI, or AI macrophage cocultures treated with IgG1 control or TGF $\beta$-blocking antibodies. Blot is representative, and quantifications are normalized to the UNP control (CTL) macrophage co-culture and shown as mean \pm SE ( $n=3$ independent experiments; Student's $t$-test).(I and J) Representative microscopy images showing a co-culture of MDA-MB-231-HRE>GFP breast cancer cells (GFP, green) and Al macrophages (red, CMTMR) in co-cultures treated with lgG control, TGF $\beta$ and IL-10 blocking antibodies. Scale bars: $50 \mu \mathrm{m}$ merged, $100 \mu \mathrm{m}$ zoom. Number of GFP positive cells per field in MDA-MB-231-HRE>GFP cell-Al macrophage co-cultures treated with IgG control, TGF $\beta$ - and IL-10-blocking antibodies ( $\mathrm{n}=3$ independent experiments, 5 fields per experiment; Student's $t$-test, ${ }^{*} P<0.05 ;{ }^{* *} P<0.01$; ns, not significant). 
A

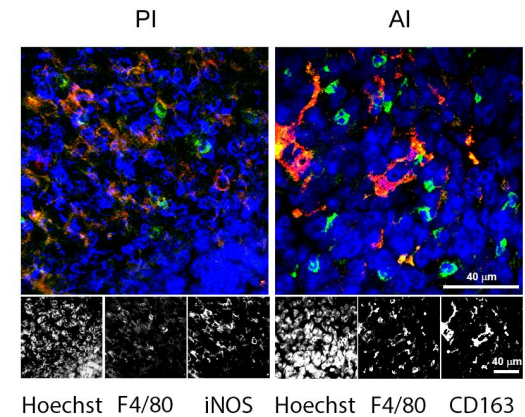

C

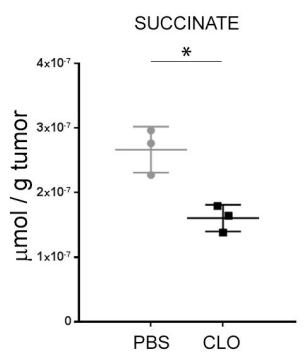

$\mathbf{F}$

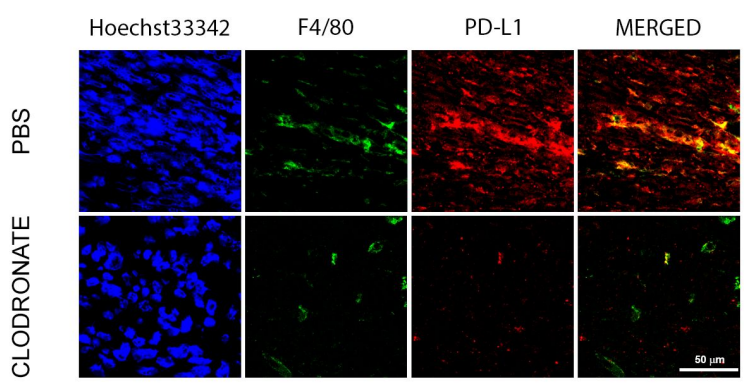

H

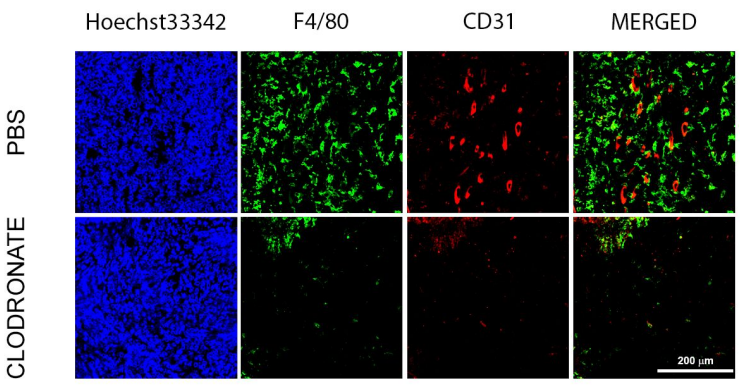

B

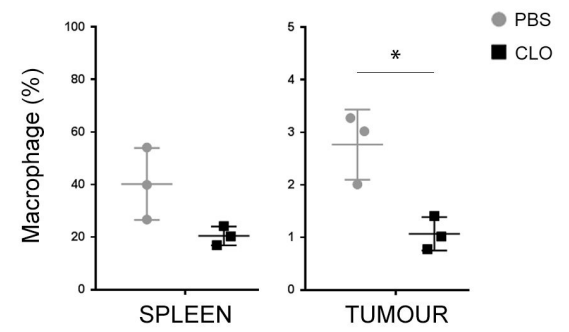

$\mathbf{E}$

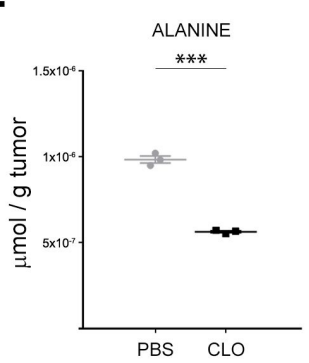

G

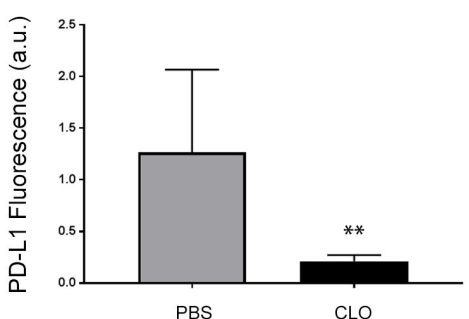

Figure 4. TAMs regulate tumor metabolism in vivo. (A) Representative images of PI $(\mathrm{F} 4 / 80+\mathrm{iNOS}+)$ and $\mathrm{Al}$ macrophages (F4/80+ CD163+) in tissue sections from control 4T1 tumors $(\mathrm{n}=3$ mice, scale bar $=40 \mu \mathrm{m})$. (B) Macrophage quantification $(\mathrm{CD} 45+$ CD11 $\mathrm{b}+$ CD127-) in spleens and tumors of 4T1 tumor-bearing mice treated with PBS or clodronate liposomes. Results are shown as a percentage of the total live cell population $(n=3$ mice; Student's $t$-test, ${ }^{*} P<0.05$ ). (C to E) Metabolite quantification by ${ }^{1} \mathrm{H}-\mathrm{NMR}$, shown as absolute 
metabolite quantification (expressed as $\mu \mathrm{mol} / \mathrm{g}$ tumor) for $(C)$ succinate, (D) lactate and (E) alanine in control (PBS) and clodronate-treated 4T1 tumor-bearing mice ( $\mathrm{n}=3$ mice; Student's $t$-test, $\left.{ }^{*} P<0.05,{ }^{* *} P<0.01,{ }^{* *} P<0.001\right)$.(F and $\left.\mathbf{G}\right)$ Representative images of PD-L1 expression (red) in tumors from PBS or clodronate-treated mice. F4/80 (green) and Hoechst33342 (blue) are used to stain macrophages and cell nuclei respectively (scale bar $=50 \mu \mathrm{m}$ ). Quantification of PD-L1 fluorescence intensity in PD-L1 ${ }^{+} \mathrm{F} 4 / 80^{-}$cells imaged in $(\mathrm{F})$ shown as mean $\pm \mathrm{SE}$ ( $\mathrm{n}=3$ mice, 5 images per mouse; Student's $t$-test, $\left.{ }^{* *} P<0.01\right)$. (H) Representative images of vascular formation (CD31, red) in tumors from PBS or clodronate-treated mice. F4/80 (green) and Hoechst33342 (blue) stain macrophages and cell nuclei respectively ( $n=3$ mice, scale bar $=200 \mu \mathrm{m})$. 
A

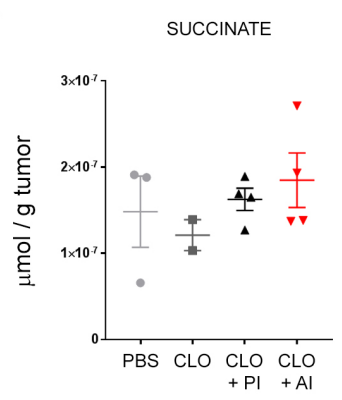

B

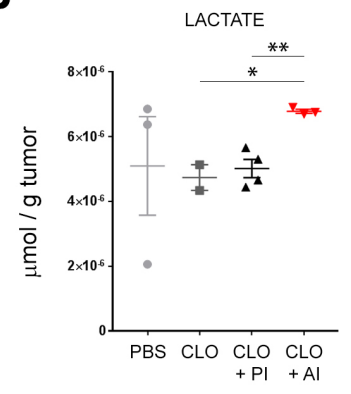

C

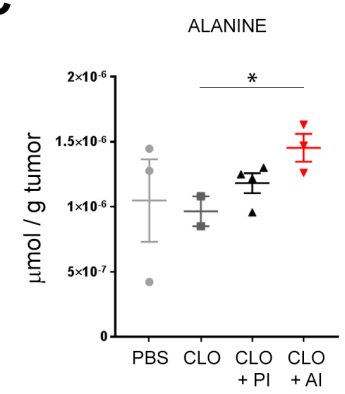

E

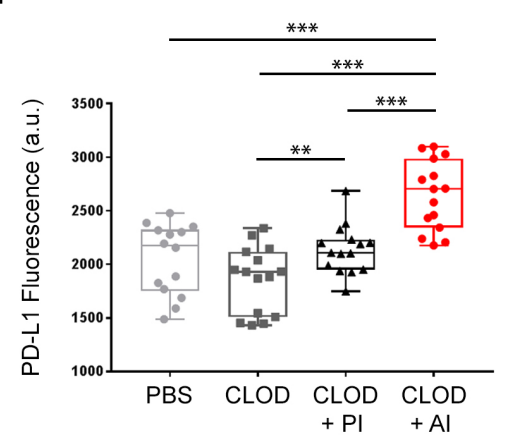

D

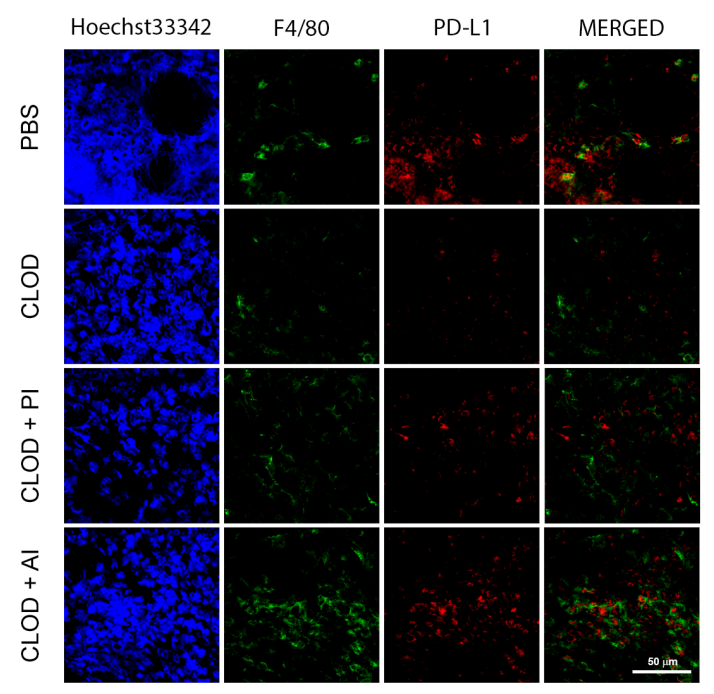

